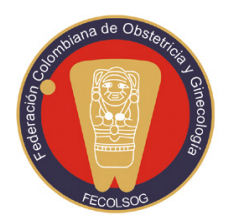

Revista Colombiana de Obstetricia y Ginecología Vol. 70 No. 2 •Abril-Junio 2019 • (103-114)

INVESTIGACIÓN ORIGINAL

DOI: https://doi.org/10.18597/rcog.3275

\title{
CUMPLIMIENTO INADECUADO DE LAS RECOMENDACIONES PARA EL PROCESO DE LA INDUCCIÓN DEL TRABAJO DE PARTO COMO DESENCADENANTE DE LA CESÁREA EN MUJERES CON EMBARAZO SIMPLE A TÉRMINO. ESTUDIO DESCRIPTIVO
}

Inadequate adlherence to the recommendlations regarding labor induction as a trigger of cesarean section in women with single, term pregnancy. A descriptive study

Elkin Alonso Álvarez-Zapata, MD ${ }^{I}$; Liza María González Hernández MD ${ }^{1}$; Nidia Beatriz Jiménez Arango $\mathrm{MD}^{1}$; John Jairo Zuleta Tobón $M D, M^{2}{ }^{2}$

Recibido: noviembre 19/18 - Aceptado: junio 18/19

\section{RESUMEN}

Objetivo: describir las características del proceso de inducción del trabajo de parto relacionadas con el exceso de cesáreas en las mujeres a quienes se les realiza este procedimiento.

Materiales y métodos: cohorte histórica descriptiva en la que se incluyeron las gestantes sin antecedente de cesárea, con embarazo único, a término y presentación cefálica, sometidas a inducción del trabajo de parto en un hospital de tercer nivel de complejidad, en Medellín, Colombia, en el periodo de mayo de 2015 a octubre de 2016. Se excluyeron mujeres con feto muerto antes de la inducción. Muestreo consecutivo. Se midieron: edad materna, paridad, edad gestacional, indicación de la inducción

* Correspondencia: John Jairo Zuleta Tobón, calle 41B Sur No. 45G-84 Envigado (Colombia).jjzuleta@une.net.co

1 Universidad de Antioquia, Medellín (Colombia).

2 Universidad de Antioquia, Medellín (Colombia). NACER, Salud Sexual y Reproductiva - Departamento de Obstetricia y Ginecología, Universidad de Antioquia, Medellín (Colombia).jjzuleta@une.net.co del trabajo de parto, favorabilidad del cérvix, tiempo de la inducción, calidad de la actividad uterina lograda, tipo de parto, momento de la inducción en que se decide la cesárea. Para definir el cumplimiento de las recomendaciones de inducción se tuvieron como referencia las guías de práctica clínica de organizaciones internacionales de la especialidad y las nuevas directrices generadas en la propuesta de reducción de la primera cesárea del año 2012. Se utilizó estadística descriptiva.

Resultados: de 2402 nacimientos se seleccionaron 289 que cumplieron con los criterios de inclusión. Se realizó cesárea al 48 \% de las gestantes, a 60,8\% de las nulíparas y a 32,1\% de las multíparas sometidas a inducción. El 72,2 \% de las que tenían cérvix desfavorable recibieron oxitocina como método de maduración cervical. A 108 (37\%) de las mujeres llevadas a inducción de parto se les realizó cesárea por diagnóstico de inducción fallida que se consideró inadecuado en todas ellas, ya que el diagnóstico 
se realizó antes de alcanzar $6 \mathrm{~cm}$ de dilatación en 88 (81,5\%), con membranas íntegras en 67 (62\%), sin actividad uterina en 42 (38,9\%), con actividad uterina de mala calidad en 23 (21,3\%) y 55 (61\%), no tuvieron al menos 24 horas de fase latente antes de realizar la cesárea.

Conclusión: se encontró falta de cumplimiento de las recomendaciones para una adecuada inducción que lleva a un diagnóstico errado de inducción fallida. Palabras clave: trabajo de parto inducido; cesárea.

\section{ABSTRACT}

Objective: To describe the characteristics of the labor induction process associated with the excess number of cesarean sections in women subjected to this intervention.

Materials and methods: Descriptive historical cohort that included pregnant women without a history of previous cesarean section, with single term pregnancy and cephalic presentation who were subjected to labor induction in a Level III complexity hospital in Medellín, Colombia, during the time period between May 2015 and October 2016. Consecutive sampling was used. Measured variables were maternal age, parity, gestational age, indication for labor induction, cervical favorability, time of induction, quality of uterine activity achieved, type of delivery, and time point during induction when the decision of cesarean section was made. The clinical practice guidelines of international organizations of the specialty and the new guides arising from the 2012 proposal of limiting the first cesarean section were used in order to define adherence to the recommendations for induction.

Results: Of the 2402 births, 289 which met the inclusion criteria were selected. Cesarean section was performed in $48 \%$ of the women subjected to induction, $60.8 \%$ nulliparous and $32.1 \%$ multiparous. Of those with unfavorable cervix, 72.2\% received oxytocin for cervical maturation. Of the women subjected to delivery induction, 108 (37\%) underwent cesarean section due to a diagnosis of failed induction. This was considered inadequate in all of them, considering that the diagnosis was made before reaching a dilatation of $6 \mathrm{~cm}$ in 88 (81.5\%), with intact membranes in 67 (62\%), with no uterine activity in 42 (38.9\%), with poor quality uterine activity in 23 (21.3\%) and in 55 (61\%) who did not have at least 24 hours of latent phase before undergoing cesarean section.

Conclusion: Failure to adhere to the recommendations for adequate induction was found, added to a mistaken diagnosis of failed induction.

Key words: Induced labor; cesarean section.

\section{INTRODUCCIÓN}

La cesárea es un procedimiento salvador de vida para el feto, para la madre o para ambos, cuando se realiza con indicaciones clínicas claras, y ha contribuido a disminuir la morbimortalidad materna y perinatal. Sin embargo, el rápido aumento de la tasa de cesáreas en las últimas décadas crea preocupación por la sobreutilización del procedimiento (1), principalmente porque se ha asociado a aumento del riesgo de complicaciones y de mortalidad materna y perinatal (2-5).

Actualmente, se registran altas tasas de cesárea en el mundo, tanto en países desarrollados como en vía de desarrollo (6). En Colombia se presentó un incremento del 24,9\% en 1998 a 45,7\% en el año 2013 (7). La situación no es diferente en el Hospital Universitario San Vicente Fundación (HUSVF) de Medellín (Colombia), en donde en un estudio que evaluó los partos entre los años 2011 y 2012, se evidenció una proporción de cesáreas del 43,1\%. Mediante la aplicación del modelo de Robson se identificó un exceso en la frecuencia de cesárea en los subgrupos de mujeres con embarazo a término y feto en cefálica, a quienes se les realizó inducción del trabajo de parto o se programó cesárea sin trabajo de parto, con respecto a otras instituciones de la región y del mundo (8). De nueve estudios evaluados que presentan información discriminada para la inducción en mujeres de estos subgrupos, grupo 2a de Robson correspondiente a las nulíparas y 4 a correspondiente a mujeres con partos previos, 
la mayor proporción de cesáreas descritas ha sido $41,5 \%$ (9) y 27,3\%, respectivamente (10).

La inducción es la estimulación artificial del trabajo de parto, antes de su inicio espontáneo, con la intención de lograr un parto vaginal. Es un procedimiento indicado cuando los riesgos de continuar el embarazo superan los riesgos asociados con el trabajo de parto inducido y el parto (11). Las técnicas modernas varían según el estado del cérvix antes de la inducción e incluyen: los agentes de maduración para el cérvix desfavorable y los métodos de inducción del parto para el cérvix favorable. Entre los primeros se incluyen la administración local de medicamentos que ablandan y dilatan el cuello uterino, como las prostaglandinas, y los métodos mecánicos, que incluyen la inserción de catéteres o dilatadores directamente en el endocérvix. La oxitocina no se recomienda como método de maduración cervical, por su efecto limitado y por la existencia de alternativas más efectivas. En cuanto a los inductores del parto, se incluyen la administración de medicamentos sistémicos que estimulan las contracciones uterinas, como la oxitocina sintética, y los métodos mecánicos como la amniotomía (12). En Estados Unidos se ha descrito una posible asociación entre la inducción del trabajo de parto y el aumento de la frecuencia de partos por cesárea, debido al incremento simultáneo y paralelo de ambos procedimientos (13); sin embargo, la verdadera relación entre ellos es contradictoria ya que varios metaanálisis han descrito más bien una disminución de la cesárea, incluso en subgrupos de mujeres con aparente mayor riesgo de ser sometidas a esta operación (14-17).

La Organización Mundial de la Salud (OMS) propone caracterizar y clasificar los eventos y los resultados en la población atendida en relación con las cesáreas en cada unidad de partos para determinar su propia tasa y analizar qué factores están influyendo en el alto porcentaje de procedimientos, para intervenirlos en busca de reducir la frecuencia $(18,19)$. El objetivo de este estudio fue describir las características del proceso de inducción del trabajo de parto y relacionar la inducción con el exceso de cesáreas previamente identificado en la institución en las mujeres a quienes se les realiza este procedimiento.

\section{METODOLOGÍA}

Diseño y población. Cohorte histórica descriptiva. Se incluyeron mujeres con inducción del trabajo de parto, con embarazo único mayor o igual a 37 semanas, con feto en cefálica, con o sin parto vaginal previo, y sin antecedente de cesárea, atendidas en un hospital general de tercer nivel de complejidad de Medellín (Colombia), que atiende población perteneciente al aseguramiento subsidiado por el Estado en el sistema general de seguridad social en salud (SGSS). Se excluyeron las mujeres con diagnóstico de feto muerto antes de la inducción. Para estimar la frecuencia de inducción bien conducida, asumiendo un $75 \%$, estimada a partir de datos no publicados de la institución, un promedio de 1500 partos al año, con un nivel de confianza del $95 \%$ y un margen de error del $5 \%$, se requerían 289 mujeres. El muestreo fue consecutivo estricto.

Procedimiento. A partir del registro sistematizado de partos que realiza de manera prospectiva el Hospital se identificaron las historias de las pacientes de la población candidata a ingresar al estudio. Tres residentes de ginecología y obstetricia revisaron, una a una, todas las historias clínicas del listado, iniciando con las de las mujeres atendidas en octubre de 2016 y de manera estrictamente consecutiva hacia atrás, se fueron ingresando al estudio los casos que cumplían todos los criterios de inclusión hasta completar el tamaño de muestra calculado en el protocolo, lo cual se alcanzó con los partos atendidos hasta mayo de 2015. Se diseñó un formato que incluyó las variables demográficas y clínicas en el cual se transcribió directamente la información consignada en la historia clínica por el equipo tratante.

Se identificó el puntaje de Bishop dado antes del inicio de la inducción; para los casos en que no se reportó de manera directa el puntaje de Bishop, 
este se calculó mediante sumatoria de los correspondientes valores, esto si el médico había descrito de manera independiente en la historia clínica todas las características necesarias para calcularlo. Cuando no se encontró una medición objetiva de alguno de los parámetros, sino descripciones cualitativas, el equipo investigador, por decisión de consenso previa, asignó 3 puntos para el cérvix borrado, 2 para cuello corto y 0 para cuello largo, y lo incluyó en el cálculo. Se definió como cérvix favorable un puntaje mayor de 6 (20). En los análisis se tuvieron en cuenta tanto las pacientes con Bishop aportado por historia clínica como el calculado por el grupo investigador.

El tiempo total de la inducción se cuantificó desde el inicio de la administración de cualquier método de inducción elegido por el equipo tratante, incluido el periodo de maduración cervical cuando se requirió, hasta el parto o la suspensión definitiva del método. Se consideró la maduración cervical como pertinente cuando se utilizaron prostaglandinas o métodos mecánicos. El uso de oxitocina para maduración cervical se consideró como no pertinente. Se descontaron las horas en las que se suspendía temporalmente el método de inducción y se consideró inducción continua cuando no hubo suspensiones temporales del método de inducción.

A partir de las órdenes médicas y los registros de enfermería, se calculó el tiempo mínimo y el tiempo máximo que transcurrió para el aumento de las dosis de oxitocina para cada mujer. Se consideró que hubo regularidad en los intervalos de modificación de la dosis cuando se verificó un patrón periódico en la evaluación y en la variación de oxitocina. El tiempo transcurrido entre la ruptura de membranas y el diagnóstico de inducción fallida se calculó desde la ruptura espontánea o artificial de las membranas hasta el momento del diagnóstico de inducción fallida dado por el médico. Se tomó como inicio de la fase activa a partir de $6 \mathrm{~cm}$ de dilatación, según las recomendaciones más actualizadas al respecto $(21$, 22). Se consideró buena actividad uterina cuando se describieron entre 3 y 5 contracciones uterinas en $10 \mathrm{~min}$, regulares, de buena intensidad, de 35 a 60 segundos de duración (23). El diagnóstico de inducción fallida fue tomado de acuerdo con el juicio médico registrado en la historia clínica. En estas pacientes se cuantificó el tiempo transcurrido sin cambios cervicales previo al diagnóstico. Se consideró inducción bien conducida cuando de manera simultánea se cumplió con los siguientes criterios: 24 horas o mas de administración de oxitocina,actividad uterina regular de mínimo cada tres minutos, presencia de ruptura de membranas de al menos 12 horas y un cérvix favorable (al menos $6 \mathrm{~cm}$ ) al momento del diagnóstico de inducción fallida.

Se definió como inadecuado diagnóstico de inducción fallida cuando la gestante en inducción no alcanzó una actividad uterina regular al menos cada tres minutos, y cambios cervicales después de un tiempo mínimo de 24 horas de administración de oxitocina, en presencia de ruptura espontánea o artificial de membranas de al menos 12 horas de duración y con un cérvix favorable, es decir, de al menos 6 puntos en el puntaje de Bishop (21, 23).

Se definió detención o prolongación del trabajo de parto cuando una mujer, con mínimo $6 \mathrm{~cm}$ de dilatación y membranas rotas, no tuvo o consiguió una mínima progresión durante 4 o más horas a pesar de una adecuada actividad uterina, o más de 6 horas en presencia de contracciones inadecuadas $(21,23)$.

Para mitigar el sesgo de información se diseñó un instrumento de fácil diligenciamiento, se evaluaron los registros electrónicos y manuales de las historias clínicas con el fin de obtener los datos de manera estandarizada.

Variables medidas: la edad, la paridad, la edad gestacional, la indicación de la inducción; la necesidad o no de maduración cervical, el método usado para la maduración cervical, la descripción de las características del cérvix y el estado de las membranas al momento del inicio de la inducción, el método utilizado para la inducción, el tiempo de inducción, el tiempo de ruptura de membranas, inducción bien conducida o uso inadecuado del diagnóstico 
de inducción fallida, el uso de analgesia epidural, la vía del parto y su instrumentación, la indicación de la cesárea y el peso y el APGAR del recién nacido.

Análisis estadístico. Las variables cualitativas se presentan como número absoluto y porcentaje. A las variables cuantitativas se les evaluó la distribución con la prueba de Kolmogorov-Smirnov y las diferentes gráficas de la evaluación de la distribución normal; las que tuvieron distribución normal se presentan con media y desviación de estándar, o como mediana y rango intercuartílico (RIC) si no tenían tal distribución. Para el análisis de los datos se utilizó el software estadístico SPSS 23.

Aspectos éticos. Este estudio contó con la aprobación del comité de ética en investigación institucional.

\section{RESULTADOS}

Se identificaron 2402 historias de los nacimientos registrados entre mayo de 2015 y octubre de 2016 y se incluyeron 289 mujeres que cumplieron con los criterios de inclusión en ese periodo.

La mediana de edad de las mujeres incluidas fue 24 años (RIC 20-31), 158 (54,6\%) eran nulíparas, 156 (53,9\%) tenían entre 37 y 38 semanas de gestación, 108 (37,3\%) entre 39 y 40, 23 (7,95\%) tenían 41 semanas y 2 (0,69\%) tenían 42 o más. La tabla 1 presenta las indicaciones de inducción de trabajo de parto; 29 (10\%) tuvieron indicaciones no reportadas en la literatura: embarazo de 40 semanas, polihidramnios, comorbilidades asociadas como VIH, epilepsia, infección urinaria recurrente, trombocitopenia gestacional, masa ovárica, anemia de células falciformes y malformaciones fetales como megacisterna magna y tetralogía de Fallot. Un total de 149 (52\%) tuvo parto vaginal espontáneo, 2 (0,7\%) tuvieron parto instrumentado y 138 (48\%) parto por cesárea. Por otra parte, 96 de las 158 nulíparas (60,8\%), grupo 2a de Robson, y 42 de las 131 multíparas (32,1\%), grupo 4a, tuvieron parto por cesárea.

En 19 (6,6\%) se encontró el valor del puntaje de Bishop consignado en la historia clínica y 86 (29,8 \%), aunque no tenían el puntaje directamente consignado en la historia clínica, contaban todos los parámetros necesarios para calcularlo. A 52 (18\%), el equipo tratante solo les asignó una calificación cualitativa, como cérvix favorable o desfavorable, y 132 (45,7\%) no tuvieron ningún dato que permitiera aproximarse a alguna clasificación. Un total de 66 $(22,8 \%)$ mujeres tuvieron criterios objetivos para considerarlas con puntaje de Bishop desfavorable, 54 de estas $(81,8 \%)$ recibieron algún método de maduración cervical y las otras $12(18,2 \%)$ recibieron oxitocina con esquema de inducción desde inicio (considerado como no pertinente). De estas mujeres con cérvix desfavorable en que se realizó maduración cervical, en 15 (27,8\%) se utilizó dinoprostona y en 39 (72,2\%) oxitocina, con dosis bajas e incrementos espaciados, lo cual no se calificó como incorrecto, a pesar de no ser el método ideal. Finalmente, 32 de las 66 mujeres (48,5\%) con cérvix desfavorable y 27 de las 39 (69,2\%) con cérvix favorable tuvieron parto vaginal. En la tabla 2 se presentan las características de la inducción y algunas características del recién nacido.

A 108 de las 289 (37\%) pacientes llevadas a inducción de parto se les realizó cesárea por diagnóstico de inducción fallida. Una mujer pudo presentar una o más de una conducta incorrecta de manera simultánea. El diagnóstico se llevó a cabo antes de alcanzar la fase activa del trabajo de parto en 85 (78,7\%). A 67 se les diagnosticó inducción fallida con membranas íntegras y a 25 de las 38 que las tenían rotas al momento del diagnóstico, se les asignó este diagnóstico antes de 12 horas de ocurrida la ruptura, es decir, en 92 (87,6\%) no se cumplió la recomendación de esperar al menos 12 horas de ruptura de membranas antes de confirmar este diagnóstico. En 3 pacientes no se anotó en la historia clínica el estado de las membranas al momento del diagnóstico de inducción fallida. Al momento del diagnóstico, en la historia clínica se identificó que 42 (38,9\%) no tenían actividad uterina, en 23 (21,3\%) se consideró que era de mala calidad y en 12 (11,1\%) no se anotó ninguna referencia sobre la calidad de la actividad uterina. Cincuenta y cinco $(61,0 \%)$ no tuvieron al menos 24 horas de inducción antes de realizar la cesárea. 


\begin{tabular}{|c|c|} 
Tabla 1. \\
$\begin{array}{c}\text { Indicaciones de inducción en mujeres gestantes a término en una institución de referencia } \\
\text { en Medellín (Colombia), } 2015-2016\end{array}$ \\
\hline Diagnóstico & n (\%) \\
\hline Trastornos hipertensivos asociados al embarazo & $122(42,2)$ \\
\hline Ruptura prematura de membranas en embarazo $\geq 37$ semanas & $45(15,5)$ \\
\hline Restricción del crecimiento intrauterino & $44(15,2)$ \\
\hline Embarazo $>41$ semanas & $23(8)$ \\
\hline Diabetes mellitus & $6(2)$ \\
\hline Enfermedad cardiaca materna & $6(2)$ \\
\hline Oligohidramnios & $6(2)$ \\
\hline Colestasis materna & $1(0,3)$ \\
\hline Sindrome antifosfolípido & $1(0,3)$ \\
\hline Inducción electiva* & $5(1,7)$ \\
\hline Indicación no reportada en la literatura & $29(10)$ \\
\hline Sin dato & $1(0,3)$ \\
\hline
\end{tabular}

*Sin indicación médica clara, por petición de la paciente u otras causas no clínicas.

La dosis máxima de oxitocina fue menor de $8 \mathrm{mU} /$ min en la mitad de las mujeres con diagnóstico de inducción fallida, ninguna llegó a la dosis máxima de $30 \mathrm{mU} / \mathrm{min}$ a pesar de no tener buena actividad uterina y algunas permanecieron con la misma dosis a pesar de no tener progresión. En ninguno de los casos de inducción fallida se cumplieron de manera simultánea todas las recomendaciones para su adecuada realización, por tanto, se catalogaron como inducciones mal conducidas. En la tabla 3 se presentan otras condiciones características de los casos considerados como inducción fallida. Si en vez de utilizar el criterio más actualizado para definir trabajo de parto activo, $6 \mathrm{~cm}$ de dilatación, se hubiera utilizado el previo, $4 \mathrm{~cm}$, la proporción de mujeres con diagnóstico de inducción fallida antes de llegar a la fase activa sería igualmente alta, 66 (61,1\%).

\section{DISCUSIÓN}

Este estudio encontró que en ninguna de las mujeres a quienes se les asignó diagnóstico de inducción falli- da se cumplieron los criterios para considerar que la inducción fue bien conducida, requisito indispensable para aceptar como válido ese diagnóstico (23). En muchos casos se utilizaron métodos de maduración cervical inadecuados, no se realizó evaluación objetiva del estado del cérvix antes del inicio de la maduración o la inducción, no se respetaron los tiempos mínimos recomendados de perfusión de oxitocina y de ruptura de membranas para declarar inducción fallida, y no se realizaron los incrementos regulares de oxitocina hasta la dosis máxima recomendada para lograr una adecuada conducción del trabajo de parto. Esto se reflejó en una alta proporción de cesáreas en las mujeres en quienes se realizó inducción del trabajo de parto y en una proporción de inducción fallida superior a lo reportado en la literatura, que fluctúa entre 20 y $30 \%$ (24).

Como consecuencia de estos hechos, la frecuencia de cesárea en este estudio es superior a la de 156 de los 157 ensayos clínicos incluidos en una revisión sistemática del tema de inducción (14), y comparable 


\section{Tabla 2.}

Proceso de inducción, métodos y esquemas en 289 mujeres gestantes a término y características del recién nacido en una institución de referencia en Medellín

(Colombia), 2015-2016

Características de la
Puntaje de Bishop al inicio de
clínica o calculado con datos extro
- 0 - 6
- $7-13$
- "Favorable"
- "Desfavorable"
- Sin dato y sin descripción

Resultado

Estado de las membranas al inicio de la inducción: n (\%)

- Íntegras

- Rotas

$66(22,8)$

$39(13,5)$

$33(11,4)$

$19(6,6)$

$132(45,7)$

- Sin dato

$234(81,0)$

$47(16,3)$

$8(2,8)$

Método usado para maduración cervical: n (\%)

- Misoprostol

$3(1,0)$

- Dinoprostona

$66(22,8)$

- Oxitocina

$154(53,3)$

- No requerido

$66(22,8)$

Método de inducción del trabajo de parto: n (\%)

- Misoprostol

- Dinoprostona

$3(1,0)$

- Oxitocina

$38(13,1)$

- Amniotomía más oxitocina.

$245(84,8)$

$3(1,0)$

Tiempo total de inducción del trabajo de parto.

Horas (mediana, percentiles 25-75)

Inducción continua: n (\%)

- Sí

$160(55,4)$

- No

$127(43,9)$

- Sin dato

$2(0,7)$

Uso de analgesia epidural: $\mathrm{n}(\%)$

- Sí

$111(38,4)$

- No

$178(61,6)$

Intervalo mínimo de cambio de dosificación de la oxitocina.

Horas (mediana y percentiles)

$2(1-4,5)$

Intervalo máximo de cambio de dosificación de la oxitocina.

Horas Mediana (percentiles 25-75)

$5(3-7)$ 


\begin{tabular}{l|c}
\multicolumn{1}{|c|}{ Características de la inducción y el recién nacido } & Resultado \\
\hline Regularidad de los intervalos de evaluación: n (\%) & $7(2,8)$ \\
$\quad$ - Sí & $207(82,8)$ \\
- No & $34(13,6)$ \\
- Sin cambio de dosis & $2(0,8)$ \\
- Sin dato & $7,66(4,65)$ \\
\hline Máxima dosis administrada de oxitocina. mU media & \\
(Desviación estándar - DS) & $108(78,3)$ \\
\hline Indicación de la cesárea: n (\%) & $13(9,4)$ \\
Inducción fallida & $11(7,9)$ \\
Desproporción cefalopélvica & $6(4,4)$ \\
Compromiso del bienestar fetal & $3071(492,3)$ \\
Otras & $8(8-9)$ \\
\hline Peso al nacer (en g). Media (DS) & $10(9-10)$ \\
\hline APGAR al minuto. Mediana (percentiles 25-75) & \\
\hline APGAR a los 5 minutos. Mediana (percentiles 25-75) & \\
\hline
\end{tabular}

con el 47,4\% obtenido en un ensayo clínico en el que se incluyeron exclusivamente mujeres con embarazo prolongado y cérvix desfavorable, es decir, mujeres con mal pronóstico de parto vaginal (25).

Una primera explicación para este exceso de cesáreas en mujeres con inducción puede ser el método seleccionado para la maduración cervical cuando se requirió, dado que el más utilizado fue la oxitocina, y las revisiones sistemáticas apoyan el uso de misoprostol (26), de prostaglandinas (27) o de los métodos mecánicos (28), y la oxitocina no hace parte de las recomendaciones de diferentes organizaciones $(20,29,30)$.

La mayor proporción de cesáreas es explicada por la inadecuada conducción de la inducción. En solo una tercera parte de las mujeres se hizo una valoración objetiva del estado del cérvix antes de iniciar la inducción. La inducción de mujeres con cérvix desfavorable disminuye la probabilidad de una inducción exitosa (31). La mayoría de las cesáreas se realizaron por diagnóstico de inducción fallida, y este se hizo antes cumplir con los criterios y el tiempo de inducción recomendados en la literatura.

La inducción del trabajo de parto se llevó cabo con oxitocina en la mayoría de las mujeres. Revisiones sistemáticas $(32,33)$ avalan la oxitocina en esquema de bajas dosis y de altas dosis como un método idóneo de inducción del trabajo de parto en presencia de cérvix favorable. Dosis de 8-12 $\mathrm{mU}$ min son las habitualmente necesarias para un adecuado trabajo de parto, con dosis máximas de 30 $\mathrm{mU} / \mathrm{min}(20,29)$, pero, para que esta sea efectiva, se debe garantizar su aplicación en perfusión continua, haciendo ajuste de la dosis cada 15 a 40 min de forma regular hasta garantizar buena actividad uterina, lo cual no se cumplió en este estudio.

Dentro de las fortalezas de nuestro estudio destacamos la revisión de todos los nacimientos en un periodo de 17 meses de forma secuencial, lo que disminuye el posible sesgo de selección y, adicionalmente, se hizo búsqueda exhaustiva de la información necesaria en las historias y sus registros complementarios. La principal fortaleza del estu- 


\begin{tabular}{|c|c|}
\hline $\begin{array}{l}\text { Nulíparas } \\
\text { Uno o más partos previos }\end{array}$ & $\begin{array}{l}74(68,5) \\
34(31,5)\end{array}$ \\
\hline $\begin{array}{l}\text { Método de maduración cervical } \\
\text { Misoprostol } \\
\text { Dinoprostona } \\
\text { Oxitocina } \\
\text { No requerían maduración }\end{array}$ & $\begin{array}{l}1(0,99) \\
27(25,0) \\
67(62,0) \\
13(12,0)\end{array}$ \\
\hline $\begin{array}{l}\text { Dosis máxima de oxitocina en pacientes con diagnóstico de inducción fallida y } \\
\text { mala o sin actividad uterina: } \mathrm{n}(\%) \\
-1-7 \mathrm{mU} / \mathrm{min} \\
-8-12 \mathrm{mU} / \mathrm{min} \\
-13-25 \mathrm{mU} / \mathrm{min} \\
-25-30 \mathrm{mU} / \mathrm{min}\end{array}$ & $\begin{array}{c}32(56,1) \\
13 / 22,8) \\
12(21,1) \\
0(0)\end{array}$ \\
\hline $\begin{array}{l}\text { Calidad de la actividad uterina al momento del diagnóstico } \\
\text { Sin actividad } \\
\text { - Mala } \\
\text { - Buena } \\
\text { - Sin dato }\end{array}$ & $\begin{array}{l}42(38,9) \\
23(21,3) \\
31(28,7) \\
12(11,1)\end{array}$ \\
\hline $\begin{array}{l}\text { Dilatación cervical al momento del diagnóstico de inducción fallida: n (\%) } \\
-0-5 \mathrm{~cm} \\
-6-10 \mathrm{~cm} \\
\text { - Sin dato }\end{array}$ & $\begin{array}{c}85(78,7) \\
15(13,8) \\
8(7,4)\end{array}$ \\
\hline $\begin{array}{l}\text { Periodo de tiempo sin cambios cervicales al momento de diagnóstico de deten- } \\
\text { ción de la dilatación o del trabajo de parto en fase activa. Horas (mediana, rango } \\
\text { intercuartílico) }\end{array}$ & $2(1,66-2,75)$ \\
\hline $\begin{array}{l}\text { Estado de las membranas al momento del diagnóstico de inducción fallida: n (\%) } \\
\text { - Íntegras } \\
\text { - Rotas } \\
\text { - Sin dato }\end{array}$ & $\begin{array}{l}67(62) \\
38(35,1) \\
3(2,77)\end{array}$ \\
\hline $\begin{array}{l}\text { Tiempo entre la ruptura de membranas y el diagnóstico de inducción fallida. } \\
\text { Horas, mediana (percentiles } 25-75 \text { ). }\end{array}$ & $7(3,3-19)$ \\
\hline
\end{tabular}

dio es que pone en evidencia una serie de factores modificables, en contraposición a la mayoría de la literatura consultada que se centra en identificar factores clínicos, sociales o demográficos, muchas veces no factibles de intervención (34), adicional a que la literatura publicada sobre la evaluación de la adecuada inducción del trabajo del parto es escasa a nivel local e internacional. Por otro lado, dentro de las debilidades está el carácter retrospectivo debido la deficiencia o a la ausencia de muchos 
datos en las historias clínicas, lo cual puede influir en la interpretación de los resultados; por ejemplo, en un porcentaje importante de pacientes $(63,6 \%)$ no fue posible conocer objetivamente el grado de maduración cervical para poder clasificar la intervención inicial como maduración o inducción; de forma similar, no es posible discriminar entre la finalización del proceso de maduración cervical y el comienzo de la inducción con cuello favorable, así que asumimos el proceso y tiempo de inducción desde el momento en que se aplicó cualquier método por primera vez. Sin embargo, al tener como objetivo la evaluación del proceso que se aplica en la actualidad en la institución, esa ausencia de información se convierte en un hallazgo relevante por intervenir.

Esta falta de datos tendría una connotación de sesgo para un estudio que tenga pretensiones inferenciales o de extrapolación a otras poblaciones, sin embargo, en este caso, se convierte en un sustento adicional a la falta de cumplimiento de protocolos que lleva a toma de decisiones incorrectas o inoportunas.

\section{CONCLUSIÓN}

El exceso de cesáreas encontrado en las mujeres sometidas a inducción del trabajo de parto en la institución podría estar relacionado con fallas en el proceso de inducción y el diagnóstico errado de inducción fallida.

\section{REFERENCIAS}

1. American College of Obstetricians and Gynecologists (College), Society for Maternal-Fetal Medicine, Caughey AB, Cahill AG, Guise J-M, Rouse DJ. Safe prevention of the primary cesarean delivery. Am J Obstet Gynecol. 2014;210(3):179-93. Disponible en: http://www.ncbi.nlm.nih.gov/pubmed/24565430 https://doi.org/10.1016/j.ajog.2014.01.026

2. Villar J, Valladares E, Wojdyla D, Zavaleta N, Carroli G, Velazco A, et al. Caesarean delivery rates and pregnancy outcomes: The 2005 WHO global survey on maternal and perinatal health in Latin America. Lancet. 2006 [visitado 2012 jul 22];367(9525):181929. Disponible en: http://www.ncbi.nlm.nih.gov/ pubmed/16753484 https://doi.org/10.1016/S01406736(06)68704-7

3. Souza JP, Gülmezoglu A, Lumbiganon P, Laopaiboon M, Carroli G, Fawole B, et al. Caesarean section without medical indications is associated with an increased risk of adverse short-term maternal outcomes: The 2004-2008 WHO Global Survey on Maternal and Perinatal Health. BMC Med. 2010 [visitado 2012 ago 10];8(1):71. Disponible en: http://www.pubmedcentral.nih.gov/articlerender.fcgi? artid $=2993644 \&$ tool $=$ pmcentrez\&rendertype $=$ abstract https: $/ /$ doi. org/10.1186/1741-7015-8-71

4. Burrows LJ, Meyn La, Weber AM. Maternal morbidity associated with vaginal versus cesarean delivery. Obstet Gynecol. 2004 [visitado 2012 Aug 10];103(5 Pt 1):907-12. Disponible en: http://www.ncbi.nlm.nih. gov/pubmed/15121564 https://doi.org/10.1097/01. AOG.0000124568.71597.ce

5. Liu S, Liston RM, Joseph KS, Heaman M, Sauve R, Kramer MS. Maternal mortality and severe morbidity associated with low-risk planned cesarean delivery versus planned vaginal delivery at term. CMAJ. 2007 [visitado 2012 Jul 31];176(4):455-60. Disponible en: http://www.pubmedcentral.nih.gov/articlerender.fcgi? artid $=1800583 \&$ tool $=$ pmcentrez\&rendertype $=$ abs tract https://doi.org/10.1503/cmaj.060870

6. Silver RM. Implications of the first cesarean: Perinatal and future reproductive health and subsequent cesareans, placentation issues, uterine rupture risk, morbidity, and mortality. Semin Perinatol. 2012;36(5):315-23. Disponible en: http://www. ncbi.nlm.nih.gov/pubmed/23009962 https://doi. org/10.1053/j.semperi.2012.04.013

7. Rubio-Romero JA, Fonseca-Pérez, Molina S, Enrique J, Buitrago Leal M, Zuleta-Tobon JJ, et al. Racionalización del uso de la cesárea en Colombia. Consenso de la Federación Colombiana de Obstetricia y Ginecología (Fecolsog) y la Federación Colombiana de Perinatología (Fecopen). Bogotá, 2014. Rev Colomb 
Obstet Ginecol. 2014;65(2):139-51. Disponible en: https://revista.fecolsog.org/index.php/rcog/article/ view/62 https://doi.org/10.18597/rcog.62

8. Zuleta-Tobón JJ, Quintero-Rincón F, Quiceno-Ceballos AM. Aplicación del modelo de Robson para caracterizar la realización de cesáreas en una institución de tercer nivel de atención en Medellín, Colombia. Estudio de corte transversal. Rev Colomb Obstet Ginecol. 2013;64(2):90-9. Disponible en: https:// revista.fecolsog.org/index.php/rcog/article/view/115

9. Scarella A, Chamy V, Sepúlveda M, Belizán JM. Medical audit using the Ten Group Classification System and its impact on the cesarean section rate. Eur J Obstet Gynecol Reprod Biol. 2011 [visitado 2013 jul 10];154(2):136-40. Disponible en: http://www. ncbi.nlm.nih.gov/pubmed/20970238 https://doi. org/10.1016/j.ejogrb.2010.09.005

10. Brennan DJ, Murphy M, Robson MS, O’Herlihy C. The singleton, cephalic, nulliparous woman after 36 weeks of gestation: Contribution to overall cesarean delivery rates. Obstet Gynecol. 2011 [visitado 2014 May 16];117(2 Pt 1):273-9. Disponible en: http:// www.ncbi.nlm.nih.gov/pubmed/2 1252739 https:// doi.org/10.1097/AOG.0b013e318204521a

11. ACOG Committee Opinion No. 764: Medically indicated late-preterm and early-term deliveries. Obstet Gynecol. 2019;133(2):e151-5. https://doi. org/10.1097/AOG.0000000000003083

12. Penfield CA, Wing DA. Labor induction techniques: Which is the best? Obstet Gynecol Clin North Am. 2017;44(4):567-82. https://doi.org/10.1016/j. ogc.2017.08.011

13. Little SE. Elective induction of labor: What is the impact? Obstet Gynecol Clin North Am. 2017;44(4):60114. https://doi.org/10.1016/j.ogc. 2017.08.005

14. Mishanina E, Rogozinska E, Thatthi T, Uddin-Khan R, Khan KS, Meads C. Use of labour induction and risk of cesarean delivery: A systematic review and metaanalysis. CMAJ. 2014;186(9):665-73. Disponible en: http://www.ncbi.nlm.nih.gov/pubmed/24778358. https://doi.org/10.1503/cmaj.130925

15. Gülmezoglu AM, Crowther CA, Middleton P, Heatley E. Induction of labour for improving birth outcomes for women at or beyond term. Cochrane database Syst Rev. 2012;(6):CD004945. Disponible en: http:// www.ncbi.nlm.nih.gov/pubmed/22696345 https:// doi.org/10.1002/14651858.CD004945.pub3

16. Saccone G, Berghella V. Induction of labor at full term in uncomplicated singleton gestations: A systematic review and metaanalysis of randomized controlled trials. Am J Obstet Gynecol. 2015;213(5):62936. Disponible en: http://www.ncbi.nlm.nih.gov/ pubmed/25882916 https://doi.org/10.1016/j.ajog. 2015.04 .004

17. Sotiriadis A, Petousis S, Thilaganathan B, Figueras F, Martins WP, Odibo AO, et al. Maternal and perinatal outcomes after elective labor induction at 39 weeks in uncomplicated singleton pregnancies: A meta-analysis. Ultrasound Obstet Gynecol. 2019;53(1):26-35. Epub 2018 Dec 7. Disponible en: http://www.ncbi.nlm. nih.gov/pubmed/30298532 https://doi.org/10.1002/ uog. 20140

18. Organización Mundial de la Salud, Human Reproduction Program. Declaración de la OMS sobre tasas de cesárea. Ginebra / Suiza; 2015. Disponible en: http://apps.who.int/iris/bitstream/10665/161444/1/ WHO_RHR_15.02_spa.pdf?ua $=1$

19. Robson M, Hartigan L, Murphy M. Methods of achieving and maintaining an appropriate caesarean section rate. Best Pract Res Clin Obstet Gynaecol. 2013;27(2):297-308. Disponible en: http://www. ncbi.nlm.nih.gov/pubmed/23127896 https://doi. org/10.1016/j.bpobgyn.2012.09.004

20. ACOG Committee on Practice Bulletins - Obstetrics. ACOG Practice Bulletin No. 107: Induction of labor. Obstet Gynecol. 2009;114(2 Pt 1):386-97. Disponible en: http:/www.ncbi.nlm.nih.gov/pubmed/19623003 https://doi.org/10.1097/AOG.0b013e3181b48ef5

21. Caughey AB, Cahill AG, Guise J-M, Rouse DJ. Safe prevention of the primary cesarean delivery. Am J Obstet Gynecol. 2014 [visitado 2014 May 1];210(3):17993. Disponible en: http://www.ncbi.nlm.nih.gov/ pubmed/24565430 https://doi.org/10.1016/j. ajog.2014.01.026

22. Harper LM, Caughey AB, Odibo AO, Roehl KA, Zhao Q, Cahill AG. Normal progress of induced labor. 
Obstet Gynecol. 2012;119(6):1113-8. Disponible en: http://www.ncbi.nlm.nih.gov/pubmed/22569121 https://doi.org/10.1097/AOG.0b013e318253d7aa

23. Spong CY, Berghella V, Wenstrom KD, Mercer BM, Saade GR. Preventing the first cesarean delivery: Summary of a joint Eunice Kennedy Shriver National Institute of Child Health and Human Development, Society for Maternal-Fetal Medicine, and American College of Obstetricians and Gynecologists Workshop. Obstet Gynecol. 2012;120(5):1181-93. Disponible en: http://www.ncbi.nlm.nih.gov/pubmed/23090537

24. Gabbay-Benziv R, Hadar E, Ashwal E, Chen R, Wiznitzer A, Hiersch L. Induction of labor: Does indication matter? Arch Gynecol Obstet. 2016;294(6):1195-201. Disponible en: http://link. springer.com/10.1007/s00404-016-4171-1 https:// doi.org/10.1007/s00404-016-4171-1

25. Herabutya Y, Prasertsawat PO, Tongyai T, Isarangura $\mathrm{Na}$ Ayudthya N. Prolonged pregnancy: The management dilemma. Int J Gynaecol Obstet. 1992;37(4):2538. Disponible en: http://www.ncbi.nlm.nih.gov/ pubmed/1350540 https://doi.org/10.1016/00207292(92)90325-D

26. Hofmeyr GJ, Gülmezoglu AM, Pileggi C. Vaginal misoprostol for cervical ripening and induction of labour. Cochrane database Syst Rev. 2010;(10):CD000941. Disponible en: http://www. ncbi.nlm.nih.gov/pubmed/12535398 https://doi. org/10.1002/14651858.CD000941.pub2

27. Thomas J, Fairclough A, Kavanagh J, Kelly AJ. Vaginal prostaglandin (PGE2 and PGF2a) for induction of labour at term. Cochrane database Syst Rev. 2014;(6):CD003101. https://doi. org/10.1002/14651858.CD003101.pub3

28. Jozwiak M, Bloemenkamp KWM, Kelly AJ, Mol BWJ, Irion O, Boulvain M. Mechanical methods for induction of labour. Cochrane database Syst Rev.
2012;(3):CD001233. Disponible en: http://www. ncbi.nlm.nih.gov/pubmed/22419277 https://doi. org/10.1002/14651858.CD001233.pub2

29. Leduc D, Biringer A, Lee L, Dy J, Clinical Practice Obstetrics Committee, Special Contributors. Induction of labour. J Obstet Gynaecol Can. 2013;35(9):84057. Disponible en: http://www.ncbi.nlm.nih.gov/ pubmed/24099451 https://doi.org/10.1016/S17012163(15)30842-2

30. World Health Organization. WHO recommendations for induction of labour. World Heal Organ. 2011;139. Disponible en: http://apps.who.int/iris/bitstream/ handle/10665/70730/WHO_RHR_11.10_eng. pdf? sequence $=1$ \&isAllowed $=y$

31. Vrouenraets FPJM, Roumen FJME, Dehing CJG, van den Akker ES a, Aarts MJB, Scheve EJT. Bishop score and risk of cesarean delivery after induction of labor in nulliparous women. Obstet Gynecol. 2005;105(4):690-7. Disponible en: http://www. ncbi.nlm.nih.gov/pubmed/15802392 https://doi. org/10.1097/01.AOG.0000152338.76759.38

32. Wei S-Q, Luo Z-C, Qi H-P, Xu H, Fraser WD. High-dose vs low-dose oxytocin for labor augmentation: A systematic review. Am J Obstet Gynecol. 2010;203(4):296-304. Disponible en: http://www. ncbi.nlm.nih.gov/pubmed/20451894 https://doi. org/10.1016/j.ajog.2010.03.007

33. Kenyon S, Tokumasu H, Dowswell T, Pledge D, Mori R. High-dose versus low-dose oxytocin for augmentation of delayed labour. Cochrane database Syst Rev. 2013;7(7):CD007201. Disponible en: http://www. ncbi.nlm.nih.gov/pubmed/23853046 https://doi. org/10.1002/14651858.CD007201.pub3

34. Schoen C, Navathe R. Failed induction of labor. Semin Perinatol. 2015;39(6):483-7. Disponible en: http:// www.ncbi.nlm.nih.gov/pubmed/26341068 https:// doi.org/10.1053/j.semperi.2015.07.013 